\title{
Clinical experience of immune checkpoint inhibitor for a metastatic jejunal cancer patient with a high tumor mutational burden and low expression of programmed death-ligand 1
}

\author{
Seo Ree Kim¹, Sang Hoon Chun ${ }^{1}$, Ji Hyun Kim², Sang-Yeob Kim³ ${ }^{3}$ Bo-In Lee ${ }^{4}$, Chan Kwon Jung ${ }^{5,6}$, Jin Hyoung Kang ${ }^{1,6,7}$ \\ ${ }^{1}$ Division of Medical Oncology, Department of Internal Medicine, College of Medicine, The Catholic University of Korea, Seoul; \\ ${ }^{2}$ Division of Gastrointestinal Surgery, Department of Surgery, Seoul St. Mary's Hospital, College of Medicine, The Catholic University of Korea, Seoul; \\ ${ }^{3}$ Asan Institute for Life Sciences, Asan Medical Center, University of Ulsan College of Medicine, Seoul; \\ ${ }^{4}$ Division of Gastroenterology, Department of Internal Medicine, ${ }^{5}$ Department of Hospital Pathology, ${ }^{6}$ Cancer Research Institute, ${ }^{7}$ Laboratory of \\ Medical Oncology, Cancer Research Institute, College of Medicine, The Catholic University of Korea, Seoul, Korea
}

\begin{abstract}
Recent data showed that DNA mismatch repair deficiency can be a predictive biomarker for a favorable response of immune checkpoint inhibitors regardless of tumor type due to give rise to high tumor mutational burden (TMB) and microsatellite instability (MSI). Loss-of-function mutations of a specific tumor suppressor gene can also lead to good response to immunotherapy. Herein, we report a case exhibiting good response to pembrolizumab in a jejunal adenocarcinoma patient with low programmed death-ligand 1 (PD-L1) expression. A 67-yearold man underwent surgical resection followed by adjuvant chemotherapy. After 10 months, he was treated with palliative chemotherapy due to hepatic and pulmonary metastases. However, palliative chemotherapy did not have any effect whatsoever. Based on genetic testing results of high TMB and high MSI in the resected primary tumor, pembrolizumab treatment was performed. After the three cycles of treatment, all metastatic lesions shrank remarkably. Considering the mechanism of immune checkpoint inhibitors, this case establishes the importance of genetic markers as TMB and MSI rather than PD-L1 expression by the prediction of their anti-tumor activities..
\end{abstract}

Keywords: Jejunal neoplasm, DNA mismatch repair, Microsatellite instability

Received: Mar 12, 2020 Revised: May 23, 2020 Accepted: May 27, 2020 Correspondence to: Jin Hyoung Kang

Division of Medical Oncology, Department of Internal Medicine, Seoul St. Mary's Hospital, College of Medicine, The Catholic University of Korea, 222 Banpo-daero, Seocho-gu, Seoul 06591, Korea

Tel: +82-2-2258-6043, Fax: +82-2-594-6043

E-mail: oncologykang@naver.com

ORCID: Seo Ree Kim (https://orcid.org/0000-0001-7461-103X), Sang Hoon Chun (https://orcid.org/0000-0002-5847-7317), Ji Hyun Kim (https://orcid.org/00000003-0210-9870). Sang-Yeob Kim (https://orcid.org/0000-0002-3724-256X), BoIn Lee (https://orcid.org/0000-0002-0218-4136), Chan Kwon Jung (https://orcid. org/0000-0001-6843-3708), Jin Hyoung Kang ((https://orcid.org/0000-00023741-5168)

Copyright @ 2020 Korean Society of Surgical Oncology

This is an Open Access article distributed under the terms of the Creative Commons Attribution Non-Commercial License (http://creativecommons.org/licenses/by-nc/4.0) which permits unrestricted non-commercial use, distribution, and reproduction in any medium, provided the original work is properly cited.

\section{INTRODUCTION}

Although the small intestine makes up $75 \%$ of the entire digestive tract and $90 \%$ of its mucosal surface area, cancer arising from the small bowel is rare, accounting for less than $5 \%$ of all malignancies in the entire gastrointestinal tract $[1,2]$. The median age at diagnosis of small bowel cancer is in the sixth decade of life and the 5 -year survival rate is $65.5 \%$ in the United States [2]. The duodenum is the most frequently involved segment, accounting for $55 \%-82 \%$ of cases, followed by the jejunum (11\%-25\%) and the ileum (7\%17\%) $[1,2]$.

Immune checkpoint inhibitors, such as pembrolizumab, nivolumab, atezolizumab, and durvalumab, targeting the regulatory pathway of T lymphocytes to enhance anti-tumor immune responses have made important clinical advances and provided new weapons against cancers [3]. Their mechanism of action is not only to directly activate the immune system to attack particular targets on tumor 
cells, but also to remove inhibitory pathways that effectively block anti-tumor $\mathrm{T}$ cell responses [4,5]. These agents can induce durable clinical responses, but there has been only a small number of patients [6]. DNA mismatch repair-deficient (dMMR) cancers are rich in lymphocyte infiltration, indicating the presence of an active host immune response [5]. In tumors harboring the dMMR gene occur large numbers of mutations, known as microsatellite instability (MSI) [5,7]. The dMMR gene has been found in colorectal cancer (4\%) and reported to achieve high response rates of anti-programmed cell ceath-1 (PD-1) inhibitors [4].

Herein, we report a patient with recurrent and metastatic jejunal cancer who had a high tumor mutational burden (TMB) and low expression of programmed death-ligand 1 (PD-L1) but dramatically responded to pembrolizumab treatment. This case was approved by the Institutional Review Board of Seoul St. Mary's Hospital (IRB No. KC18SESI0440) and received informed consent from the patient.

\section{CASE REPORT}

In May 2017, a 67-year-old man visited Seoul St. Mary's Hospital due to a stomach ache. Vital signs were stable but direct tenderness was observed in right upper quadrant at initial physical examination. Abdomen computed tomography (CT) scan and positron emission tomography (PET)-CT was done for further evaluation. Small bowel tumor with enhancing wall thickening in proximal jejunum and sin- gle metastatic nodule in the right lobe of liver was observed. For pathologic confirmation, segmental resection of the small bowel was performed with the laparoscopic technique. The pathologic diagnosis was poorly differentiated adenocarcinoma with several regional lymph node metastases, thus the final staging was determined to be pT3N2M1 (stage IV). After the surgery, tomotherapy (40 Gy/4 fx) on the single metastatic lesion at the $\mathrm{S} 6$ segment of the liver was given in June 2017. Adjuvant cytotoxic chemotherapy including intravenous irinotecan $\left(180 \mathrm{mg} / \mathrm{m}^{2}\right)$, infusional 5-fluorouracil (5-FU) $\left(2,800 \mathrm{mg} / \mathrm{m}^{2}\right)$, and leucovorin $\left(400 \mathrm{mg} / \mathrm{m}^{2}\right)$ was then given on day 1 and then every 2 weeks. It was repeatedly administered for six cycles from June 2017 to December 2017.

In March 2018, a follow-up abdomen CT scan and liver magnetic resonance imaging revealed the progression of multiple liver metastases that were histologically proven as metastatic adenocarcinoma. After the positive result of polymerase chain reaction on mutations of mismatch repair (MMR) genes, we requested sequencing of target genes by next generation sequencing (NGS) which was assigned to Foundation Medicine in the United States. Meanwhile, he received two cycles of salvage chemotherapy including intravenous oxaliplatin $\left(130 \mathrm{mg} / \mathrm{m}^{2}\right.$, day 1$)$ and capecitabine $\left(1,500 \mathrm{mg} / \mathrm{m}^{2}\right.$ bid orally, day $\left.1-14\right)$ between March and April 2018. Follow-up lung and abdomen CT scans showed new lung metastases with mediastinal lymphadenopathy and progression of multiple liver metastases.

With careful interpretation of the NGS data, we decided to treat

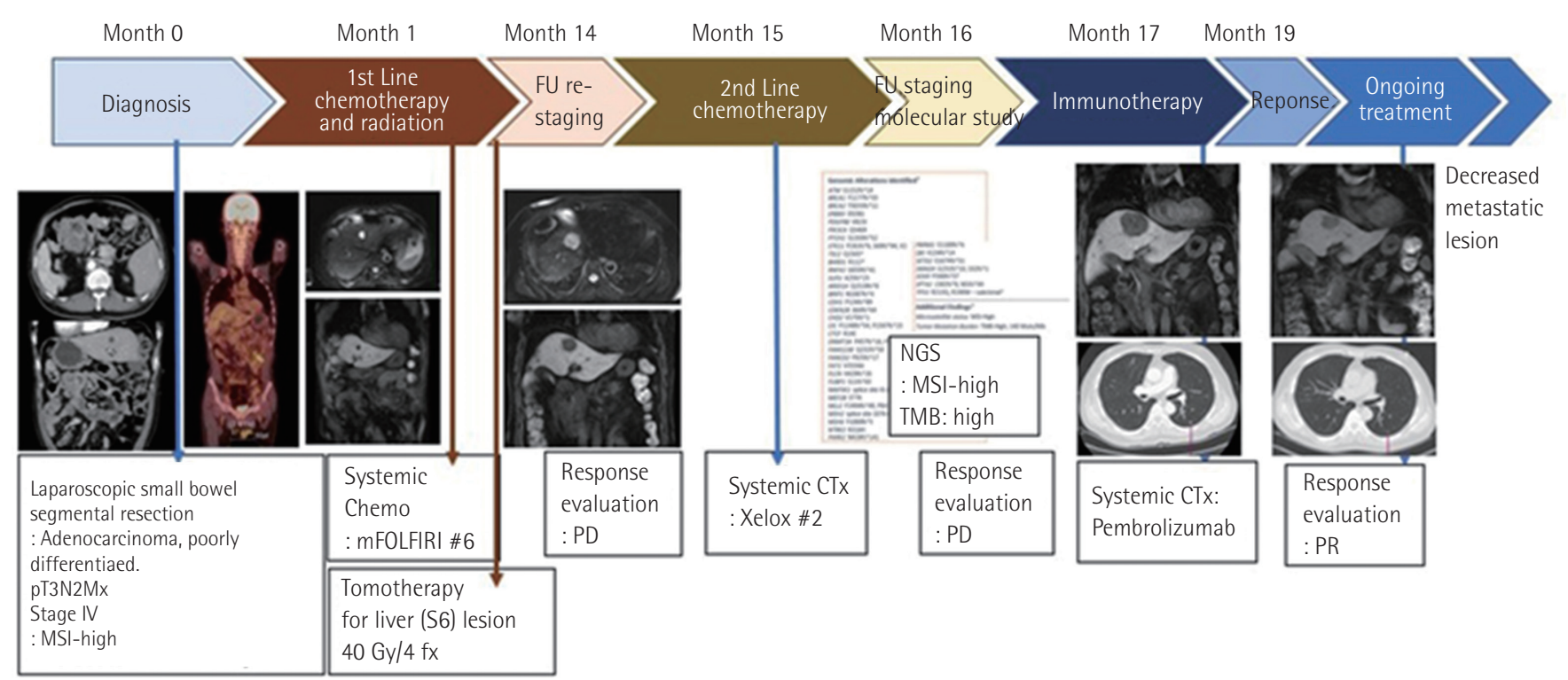

Fig. 1. Time flow regarding his treatment history and response evaluation. All metastatic lesions at liver and lung were decreased by $30 \%$ after three cycles of immunotherapy. FU, follow-up; NGS, next generation sequencing; MSI-high, microsatellite instability high; TMB, tumor mutational burden; mFOLFIRI, modified 5-fluorouracil+irinotecan+leucovorin; PD, progressive disease; CTx, chemotherapy; PR, partial response. 


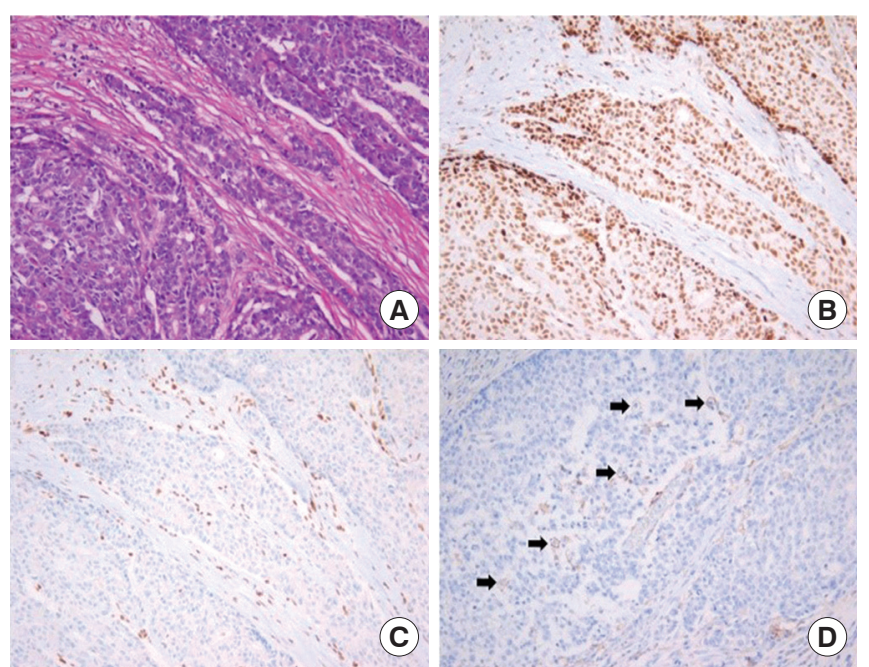

Fig. 2. Morphology and immunohistochemistry of jejunal cancer. (A) The tumor is composed of poorly differentiated adenocarcinoma (HEtE, $\times 200)$. Immunohistochemistry shows diffuse strong positive expression of MLH1 $(B, \times 200)$ and complete loss of MSH2 expression $(C, \times 200)$ in the tumor cell. (D) Programmed death-ligand 1 (PD-L1) testing using the clone $22 \mathrm{C} 3$ pharmDX shows weak membrane staining in some tumor cells (arrows; $\times 200$ ).

with pembrolizumab ( $3 \mathrm{mg} / \mathrm{kg}$ intravenously every 3 weeks) in May 2018. After the three cycles of pembrolizumab, metastatic lesions of the liver and lung were markedly decreased in size by more than 30\%, indicating partial response (Fig. 1).

\section{Genetic analysis and immune cell composition by multiplex immunohistochemistry}

The tumor tissue obtained from small bowel resection was utilized for molecular genetic study. Comprehensive genomic profiling analysis using a targeted NGS panel (FoundationOne test; Foundation Medicine, Cambridge, MA, USA) identified 50 genomic findings and 140 mutations/Mb, which means MSI-high (MSI-H) and TMB high (TMB-H). We identified innumerable frame-shift mutations in various oncogenes and tumor suppressor genes which can produce entirely foreign peptide sequences that might be immunogenic. Immunohistochemistry (IHC) staining revealed positive for MLH1, negative for MSH2, and weak membrane staining with PD-L1 (clone 22C3) in 2\% of tumor cells (Fig. 2).

We used a quantitative multispectral imaging method using Opal Multiplex IHC kit (PerkinElmer, Waltham, MA, USA) and Vectra automated quantitative pathology imaging system (PerkinElmer) to examine the microenvironment of tumor tissue obtained by the surgical specimen. The multiplex IHC staining of several immune cells, co-inhibitory signal, and PD-1/PD-L1 molecules were performed using a Leica Bond Rx Automated Stainer
(Leica Biosystems, Newcastle, UK).

To estimate the $\mathrm{T}$ cell composition, we analyzed the expression of CD4, CD8, FoxP3, CD45RO, and PD-L1 with panel A. To evaluate the density of NK cell, dendritic cell, and macrophage, which is other immune cells, we examined the degree of penetration of CD56, CD11c, CD68, and CD206 with panel B. The expressions of TIM3, LAG3, and PD-1, the co-inhibitory signal of PD-1/PDL1, were also examined using panels C, D (Fig. 3).

We found that staining was lining along the peripheral margin of the tumor, except for the stroma. We divided the whole area of tumor tissue into multiple sections and analyzed them in detail. The PD-L1 expression in the stroma was seen only in its marginal area, though too low overall (Fig. 4).

\section{DISCUSSION}

We report a metastatic jejunal cancer patient who had a favorable response to pembrolizumab that consisted of dMMR, MSI-H, and TMB-H tumor.

PD-L1 expression has been widely used as a predictive biomarker for the efficacy of PD-1/PD-L1 blockades. This case provides an intriguing paradox where PD-L1 expression in surgically resected tumor tissue is very low (2\%); it was therefore difficult to understand why this patient displayed a fairly good response despite low PD-L1 expression. There are several possible explanations for this fairly good response to pembrolizumab despite low PD-L1 expression. First, PD-L1 expression is not a prerequisite for anti-PD-1/ PD-L1 inhibitors due to its variability. Second, neoantigens arising from somatic mutations may directly elicit tumor-specific immune responses. The more mutated the tumor, the more likely it will trigger a response by preexisting $\mathrm{T}$ cells, thus evoking anti-tumor effect by anti-PD-1 blockers [8]. On the contrary, even PD-L1-positive tumors would not respond to anti-PD-1/PD-L1 therapy if it does not contain $\mathrm{T}$ cells. It is one of the possible reasons why low PD-L1 expression should not be interpreted as an unfavorable predictor for response to anti-PD-1 inhibitors. Hellmann et al. [9] have reported that PD-L1 expression levels are not correlated with the clinical outcome of anti-PD-1 blockades. Third, we should consider several limitations of IHC staining. For example, non -specific unstained and intra-tumoral heterogeneity of PD-L1 expression might be obstacles for precise assessment.

One possible explanation is that PD-1 blockade has potent anti-tumor activity for MMR-deficient cancer patients. DNA dMMR cancers are rich in lymphocyte infiltration, indicating the presence of an active host immune response [4,5]. A proposed explanation for the activity of immune checkpoint inhibitor in tumors is that it can boost T cell reactivity against "neoantigens," i.e., T cell epi- 

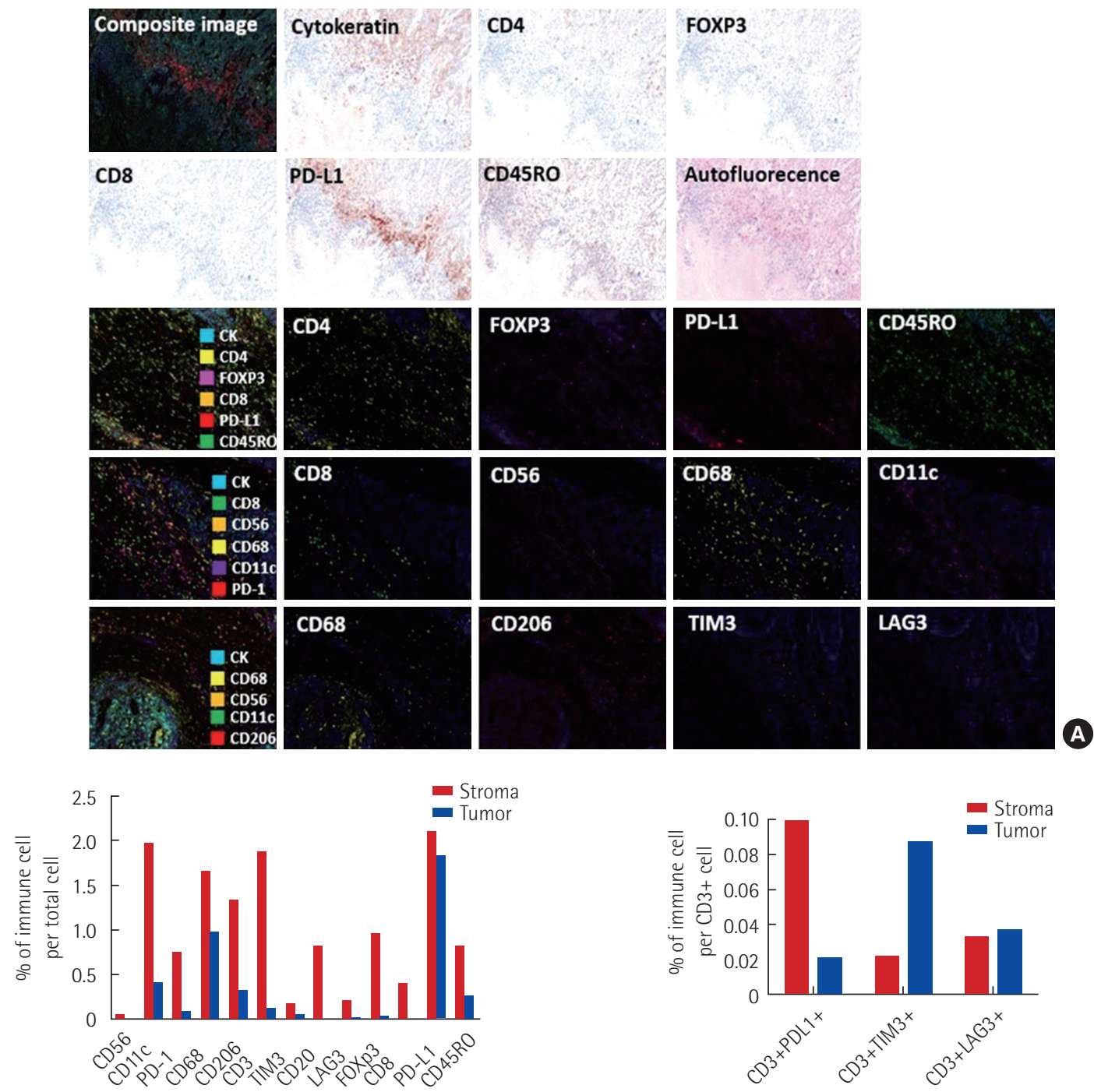

P1: CD4 (T helper), FoxP3 (T reg), CD8 (T cyto), PD-L1, CD45R0

P2: CD56 (Dendritic cell), CD11c (NK cell), PD1

P2: CD68 (pan-macrophage), CD206 (M2 macrophage)

P2: CD3 Tim3 CD20 (B cell), LAG3

Fig. 3. Multiplex immune-histological feature of small bowel resection tissue. (A) This technique provides quantitative analysis data through multiple biomarker staining, scanning and machine learning. Immunohistochemical staining with cytokeratin, CD4, CD8, CD45RO, FOXP3, PD-L1, CD56, CD11C, CD68, CD206, PD-1, CD3, CD20, TIM3, and LAG3 was performed in the same tissue. (B) The tumor tissue of the patient was well expressed in co-stimulators that formed tumor-reactive T cells and was able to activate the effector $\mathrm{T}$ cell function, and the expression of the co-inhibitory receptor or immune-suppressive cells was low.

topes that are newly formed as a consequence of tumor-specific mutations [3]. In the absence of functional MMR, original DNA strand slippage goes unrepaired, producing frame-shift mutations resulting in truncated protein containing a mutation associated neoantigen [7]. Hence, in tumors harboring the dMMR gene, several mutations can occur, such as marking on an answer sheet that is serially misplaced. Ultimately, these sequential events bring about large numbers of mutations, particularly in regions with repetitive DNA sequences, a phenomenon known as MSI [5,7]. Be- cause dMMR is incapable of preventing proto-oncogenes or tumor suppressor genes from having frame-shift mutation during transcription, this aberrant process can lead to MSI-H [4]. This situation bears a higher rate of nonsynonymous single nucleotide polymorphisms, leading to increased numbers of neoepitopes and tumor-infiltrating PD-1+ cytotoxic T lymphocytes displaying a greater response to immune checkpoint blockades (ICBs) than MSI-low or microsatellite stable cancers [4,5]. This is one of the possible explanations as to why PD-1 blockade has potent anti-tu- 
ROI region-MSI scan (x20)
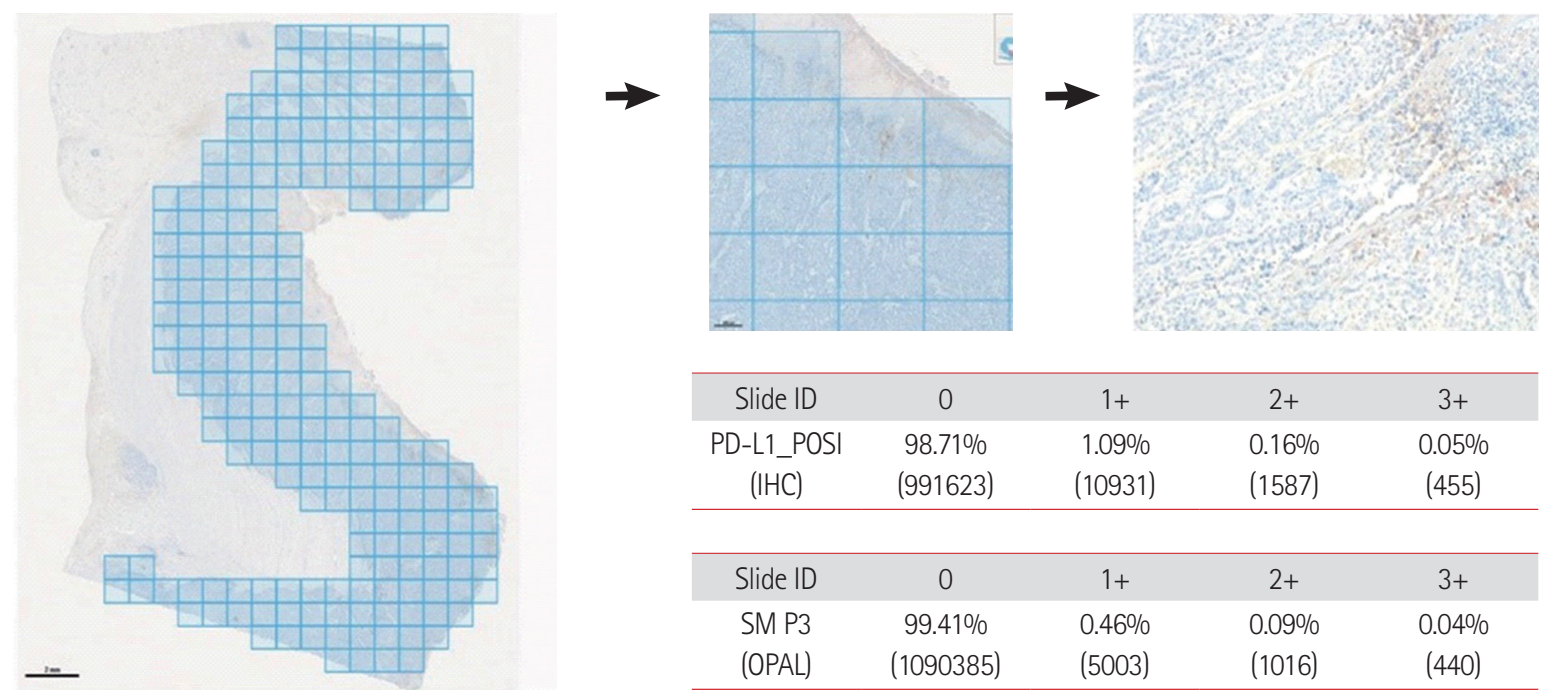

\begin{tabular}{|c|c|c|c|c|}
\hline Slide ID & 0 & $1+$ & $2+$ & $3+$ \\
\hline $\begin{array}{c}\text { PD-L1_POSI } \\
(\mathrm{IHC})\end{array}$ & $\begin{array}{c}98.71 \% \\
(991623)\end{array}$ & $\begin{array}{c}1.09 \% \\
(10931)\end{array}$ & $\begin{array}{l}0.16 \% \\
(1587)\end{array}$ & $\begin{array}{c}0.05 \% \\
(455)\end{array}$ \\
\hline Slide ID & 0 & $1+$ & $2+$ & $3+$ \\
\hline $\begin{array}{l}\text { SM P3 } \\
\text { (OPAL) }\end{array}$ & $\begin{array}{c}99.41 \% \\
(1090385)\end{array}$ & $\begin{array}{l}0.46 \% \\
(5003)\end{array}$ & $\begin{array}{l}0.09 \% \\
(1016)\end{array}$ & $\begin{array}{c}0.04 \% \\
(440)\end{array}$ \\
\hline
\end{tabular}

Fig. 4. Analysis of the whole slide was divided into multiple sections to check the extent of PD-L1 expression. ROI, region of interest; MSI, microsatellite instability; PD-L1, programmed death-ligand 1; IHC, immunohistochemistry.

mor activity for MMR-deficient cancer patients in the aspect of molecular genetics.

In recent clinical studies, Le et al. [10] have shown that cancer patients harboring dMMR are particularly responsive to ICBs. In a phase II study, the remarkable activity of PD-1 blockade was worthy of notice in 20 MMR-deficient colorectal cancer and other cancers patients compared with 21 patients with MMR-proficient colorectal cancer [10]. Thereafter, large-scale clinical studies have also compared the degree of response to ICB for advanced cancer patients with dMMR across 12 different tumor types [5]. Good responses to PD-1 blockades have been achieved in various types of dMMR advanced cancers; however, their proportion is not that high in real-world clinical practice.

Generally, anti-cancer immunity in humans is categorized into three main phenotypes: immune-desert, immune-excluded, and inflamed phenotype [9]. We quantitatively measured numbers of immune cells, including regulatory $\mathrm{T}$ cells, myeloid-derived suppressor cells, tumor-infiltrating lymphocyte (TIL)s, and cancer-associated macrophages using the multiplex IHC. Based on TIL and T cell composition in tumor microenvironment (TME), the immune phenotype of our case was classified into the immune desert $[11,12]$. As a result, the degree of immune cell penetration in the tumor did not inflame as expected. Infiltrated and inflamed TME with cytotoxic T lymphocytes and tumor-associated macrophages in the tumor margin rather than the tumor core were scattered only a little. Binnewies et al. [12] have insisted that the lack of T cell infiltration corresponds to a "desert"-like immune landscape or "cold tumor" which has low immunogenic status. In the present case, several possible explanations could be drawn about the favorable response to pembrolizumab. First, there may be many possible candidates as neoantigen to consolidate the reaction with ICB, which were produced by dMMR with MSI-H. Second, we paid attention to the loss-of-function of PBRM1 gene in the mutant dielectrics obtained from NGS. Recently, Miao et al. [13] have presented that loss-of-function due to somatic mutation of PBRM1 subunit, a tumor suppressor gene, was present in high frequency by analyzing good responders among metastatic renal cell carcinoma patients receiving immunotherapy using whole exon sequencing. They also revealed strong enrichment of immunostimulatory genes, including JAK-STAT signaling associated molecules in PBRM1-deficient cells. In this case, loss-of-function of PBRM1 functioning chromatin remodeling may play an important role in favorable response to ICB treatment. Third, the induction of tumor-driven or host-driven evolution through ICB administration could lead to a favorable response to immunotherapy. Xu-Monette et al. [14] have observed a change of PD-1/PD-L1 expression in tumor settings as a marker of $\mathrm{T}$ cell activation and driver of $\mathrm{T}$ cell dysfunction. Through host-driven innate immunogenic pathway, antigen-specific CD8+ T cells can secrete interferon- $\gamma($ IFN- $\gamma$ ) that may turn PD-L1- tumors into PD-L1+ tumors and then infiltrate PD-L1+ macrophages, dendritic cells, and T cells [14]. On the other hand, in tumor-driven evolution, oncogenic PD-L1 expression in the tumor might lead to T cell dysfunction. In immunogenic tumors, PD-L1 expression is heterogeneous. IFN- $\gamma$, a mediator of this pathway, functions oppositely, resulting in $\mathrm{T}$ cell exhaustion and eventually triggering a vicious cycle of immune suppression. 
There are several emerging tissue- and serologic-based predictive biomarkers for tumor response of ICBs beyond PD-L1 expression. Candidate biomarkers are comprehensive of TMB, cytotoxic CD8+ T cells/TILs, immune score by the density of CD8+ T cells and CD3+ T cells, multiplex IHC, T cell receptor clonality, immune gene signature, and neutrophil-to-lymphocyte ratio [15]. In our case, to make up for inaccurate predictability of PD-L1 expression, we attempted to apply several potential predictors including TMB, immune cell composition in TME with a degree of immune cell penetration using multiplex IHC.

In summary, we experienced an interesting case of a dMMR, metastatic small bowel adenocarcinoma patient carrying MSI-H and TMB-H with loss-of-function of PBRM1, displaying an excellent response to the anti-PD-l inhibitor.

\section{CONFLICT OF INTEREST}

No potential conflict of interest relevant to this article was reported.

\section{ACKNOWLEDGMENTS}

The authors wish to acknowledge the financial support obtained from the Catholic Medical Center Research Foundation made in the program year of 2018 .

\section{REFERENCES}

1. Zhang MQ, Chen ZM, Wang HL. Immunohistochemical investigation of tumorigenic pathways in small intestinal adenocarcinoma: a comparison with colorectal adenocarcinoma. Mod Pathol 2006;19:573-80.

2. Aparicio T, Zaanan A, Svrcek M, Laurent-Puig P, Carrere N, Manfredi S, et al. Small bowel adenocarcinoma: epidemiology, risk factors, diagnosis and treatment. Dig Liver Dis 2014;46:97-104.

3. Schumacher TN, Schreiber RD. Neoantigens in cancer immunotherapy. Science 2015;348:69-74.

4. Le DT, Durham JN, Smith KN, Wang H, Bartlett BR, Aulakh LK, et al. Mismatch repair deficiency predicts response of solid tumors to PD-1 blockade. Science 2017;357:409-13.

5. Kelderman S, Schumacher TN, Kvistborg P. Mismatch repair-deficient cancers are targets for anti-PD-1 therapy. Cancer Cell 2015;28:11-3

6. Cortes-Ciriano I, Lee S, Park WY, Kim TM, Park PJ. A molecular portrait of microsatellite instability across multiple cancers. Nat Commun 2017;8:15180.

7. Dudley JC, Lin MT, Le DT, Eshleman JR. Microsatellite instability as a biomarker for PD-1 blockade. Clin Cancer Res 2016;22:81320.

8. Yarchoan M, Hopkins A, Jaffee EM. Tumor mutational burden and response rate to PD-1 inhibition. N Engl J Med 2017;377:2500-1.

9. Hellmann MD, Ciuleanu TE, Pluzanski A, Lee JS, Otterson GA, Audigier-Valette C, et al. Nivolumab plus ipilimumab in lung cancer with a high tumor mutational burden. $\mathrm{N}$ Engl J Med 2018;378:2093-104.

10. Le DT, Uram JN, Wang H, Bartlett BR, Kemberling H, Eyring AD, et al. PD-1 blockade in tumors with mismatch-repair deficiency. $\mathrm{N}$ Engl J Med 2015;372:2509-20.

11. Voong KR, Feliciano J, Becker D, Levy B. Beyond PD-L1 testing-emerging biomarkers for immunotherapy in non-small cell lung cancer. Ann Transl Med 2017;5:376.

12. Binnewies M, Roberts EW, Kersten K, Chan V, Fearon DF, Merad $\mathrm{M}$, et al. Understanding the tumor immune microenvironment (TIME) for effective therapy. Nat Med 2018;24:541-50.

13. Miao D, Margolis C, Martini D, Mullane SA, Cullen D, Horak C, et al. Loss-of-function of PBRM1 to predict response to anti-PD-1/ PD-L1 therapy in metastatic renal cell carcinoma. J Clin Oncol 2017;35(15_suppl):3016.

14. Xu-Monette ZY, Zhang M, Li J, Young KH. PD-1/PD-L1 blockade: have we found the key to unleash the antitumor immune response? Front Immunol 2017;8:1597.

15. Kiriu T, Yamamoto M, Nagano T, Hazama D, Sekiya R, Katsurada $\mathrm{M}$, et al. The time-series behavior of neutrophil-to-lymphocyte ratio is useful as a predictive marker in non-small cell lung cancer. PLoS One 2018;13:e0193018. 